\title{
Bioluminescence spectra of click beetle luciferases in higher plant cells
}

\author{
Rieko Ogura ${ }^{1}$, Naoko Matsuo ${ }^{2}$, Kazuyuki Hiratsuka ${ }^{2, *}$ \\ ${ }^{1}$ Venture Business Laboratory, Yokohama National University, Yokohama, Kanagawa 240-8501, Japan; \\ ${ }^{2}$ Graduate School of Environment and Information Sciences, Yokohama National University, Yokohama, Kanagawa \\ 240-8501, Japan \\ *E-mail: hiratsk@ynu.ac.jp Tel \& Fax: +81-45-339-4413
}

Received April 11, 2011; accepted August 4, 2011 (Edited by M. Sekine)

\begin{abstract}
Reporter genes are often used to monitor gene expression in various organisms. To investigate the use of a dualcolor luciferase reporter assay (DCRA) for monitoring expression of multiple genes in higher plants, we characterized the bioluminescence and the spectra of click beetle luciferases in planta. The DCRA consists of click beetle green luciferase (CBG68luc) and click beetle red luciferase (CBRluc). The green and the red bioluminescence from CBG68luc and CBRluc, respectively, are suitable for nondestructive in vivo assays because luminescence signals can be monitored selectively using optical filters. Using transgenic plants and a transient expression system, we introduced and expressed CBG68luc and CBRluc reporter genes in plant cells and obtained luminescence spectra by spectrometry. The spectral patterns from the transgenic plant cells were similar to those from E. coli transformed with the same reporter genes. Although plant tissues contain pigments and autofluorescent materials, our data suggest that the presence of these do not interfere with gene expression monitoring by DCRA, which is therefore applicable to monitoring the regulation of gene expression in plants.
\end{abstract}

Key words: Click beetle luciferase, reporter gene, spectrum analysis, transgenic plants, transient assay.

Reporter genes are widely used as powerful tools to monitor gene expression and gene function in various organisms. Among these reporters, the firefly luciferase gene is thought to be suitable for quantitative determination of the level of gene expression, because its sensitivity and range of linear response are superior to other reporter genes such as green fluorescent protein and $\beta$-glucuronidase (Naylor 1999). However, for accurate quantification of gene expression, the simultaneous use of a reference reporter gene is needed as an internal control. The dual luciferase reporter assay (DLRA) system (Promega, Madison, WI, USA) has been developed and used widely for this purpose. This system allows quantitation of the luminescence levels of firefly luciferase (Fluc) and Renilla luciferase (Rluc) independently in a single tube (Matsuo et al. 2001; Ono et al. 2004; Sherf et al. 1996). To monitor expression of multiple genes in vivo, multicolor bioluminescence reporter systems have been developed. The dual-color luciferase reporter assay (DCRA), consisting of green luciferase (CBG68luc) and red luciferase (CBRluc) originating from the luminous click beetle, Pyrophorus plagiophthalamus, has been commercially released and used for in vivo gene expression assays in mammalian cells (Almond et al. 2003).

The Tripluc Multi Reporter Assay System has been developed to monitor the bioluminescence level of three different-color luciferases independently. This system allows monitoring of in vivo gene expression in mammalian cells and has been applied to clock gene studies (Nakajima et al. 2004a; Nakajima and Ohmiya 2010). An assay system combining these luciferases with Rluc has also been developed (Gammon et al. 2006; Nakajima et al. 2004b).

In higher plants, the Fluc system for in vivo monitoring of gene expression has been used widely for many years; on the other hand, applications of multicolor luciferase systems have not been extensively investigated. One of our previous studies showed that the DCRA system can be applied to in vivo monitoring of transient gene expression in higher plants (Ogura et al. 2005).

In this study, in order to develop the DCRA assay system in stable transgenic plants, we investigated the bioluminescence characteristics of DCRA in planta. Expression vectors for CBG68luc and CBRluc were constructed based on plasmid vectors used for transient assays in plant cells (Ogura et al. 2005). The promoter-

Abbreviations: CaMV, cauliflower mosaic virus; CBG68luc, click beetle green luciferase; CBRluc, click beetle red luciferase; DCRA, dual-color luciferase reporter assay; DLRA, dual luciferase reporter assay; Fluc, firefly luciferase; Nos, nopaline synthase; Rluc, Renilla luciferase

This article can be found at http://www.jspcmb.jp/

Published online September 20, 2011 
terminator cassette from $35 \mathrm{~S}:$ : CBG68luc-Nos or $35 \mathrm{~S}:$ : CBRluc-Nos was excised by digesting these plasmids with HindIII and EcoRI and then cloning the cassette into the HindIII-EcoRI sites of the binary vector pBI121. The binary vector was introduced into Agrobacterium tumefaciens LBA4404 by electroporation using Gene Pulser Xcell, (Bio-Rad, Hercules, CA, USA), and then introduced into Arabidopsis thaliana (ecotype Columbia) and Nicotiana tabacum cv. SR1 by Agrobacteriummediated transformation. The $\mathrm{T}_{4}$ generation of the transgenic Arabidopsis was grown on 1/2 MS medium (Murashige and Skoog 1962) at $22^{\circ} \mathrm{C}$ under continuous light $\left(50 \mu \mathrm{mol} \mathrm{m}^{-2} \mathrm{~s}^{-1}\right)$. An aqueous solution of $0.2 \mathrm{mM}$ D-luciferin potassium salt (Promega) was sprayed onto the transgenic Arabidopsis and bioluminescence was observed using an Image intensifier unit C8600 (Hamamatsu Photonics, Hamamatsu, Japan) for $10 \mathrm{~min}$ (Figure 1A-D). We detected not only all wavelength ranges (Figure 1B) but also specific wavelength ranges of bioluminescence originating from the transgenic Arabidopsis with the options of a band pass HQ560/80 m filter (Chroma Technology, Bellows Falls, VT, USA; Figure 1C) and a band pass HQ645/75 m filter (Chroma Technology; Figure 1D). Wasabi software (Hamamatsu Photonics) was used to quantify bioluminescence levels. Although, we could observe discrete bioluminescence from transgenic Arabidopsis plants, the luminescence activity of CBRluc was weaker than CBG68luc.

The $\mathrm{T}_{2}$ generation of the transgenic tobacco was grown on MS medium at $26^{\circ} \mathrm{C}$ in continuous light $(50$ $\mu \mathrm{mol} \mathrm{m} \mathrm{s}^{-1}$ ) and then bioluminescence was detected for $10 \mathrm{~min}$ as described above. Similarly to the case with Arabidopsis seedlings, we could observe discrete color of the green luminescence using a $520-600 \mathrm{~nm}$ band pass filter (HQ560/80 m), but the separation of the red luminescence using a $607-682 \mathrm{~nm}$ band pass filter (HQ645/75 m) was hampered by overlapping luminescence from CBG68luc (Figure 1F-I). In multiple lines of transgenic tobacco, we observed that the luminescence of CBRluc was considerably lower than that of CBG68luc (data not shown).

To investigate the relative luminescence of CBG68luc and CBRluc, we compared the luminescence activity of both reporters using a transient assay in Arabidopsis and tobacco. As an internal control, $35 \mathrm{~S}::$ hRluc plasmids were co-introduced into mature leaves with $35 \mathrm{~S}:$ : CBG68luc or $35 \mathrm{~S}::$ CBRluc. To make the $35 \mathrm{~S}::$ hRluc plasmid, the hRluc sequence was amplified by PCR with forward primer 5'-GGTCTAGACCATGGCTTCCAAGGTGTACGA-3' and reverse primer 5'-GGGAGCTCTTACTGCTCGTTCTTCAGCA-3' using pGL4.70 (Promega) as template. The PCR product was digested with $X b a \mathrm{I}$ and $S a c \mathrm{I}$, and then cloned into the $X b a \mathrm{I}-S a c \mathrm{I}$ sites of pBI221. The microprojectile bombardment was carried out using a model PDS-1000/He particle delivery

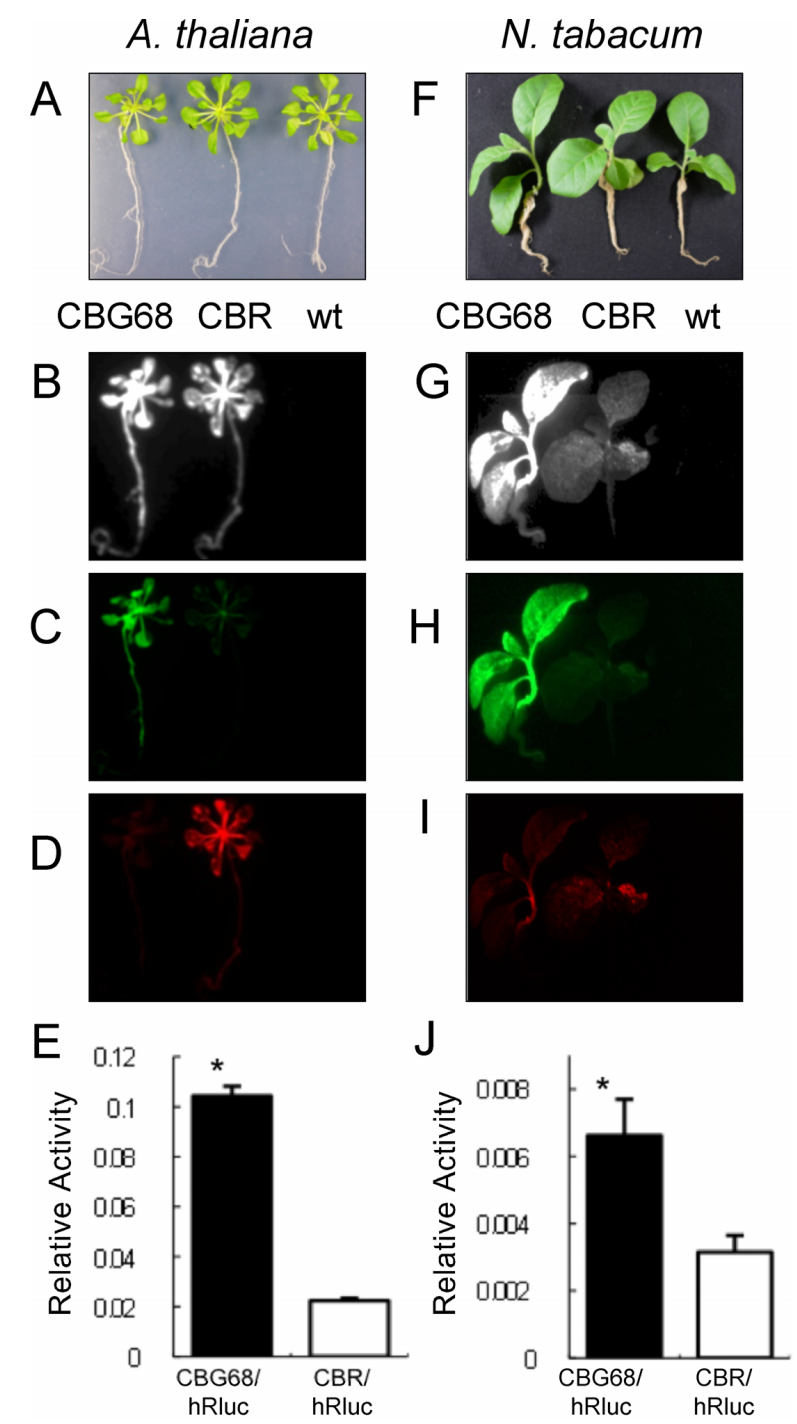

Figure 1. Activities of CBG68luc and CBRluc in transgenic Arabidopsis and tobacco. CBG68luc and CBRluc activities under the control of CaMV 35S promoter in Arabidopsis thaliana (A-D) and Nicotiana tabacum (F-I). D-luciferin was sprayed and then monitored their bioluminescence under $\mathrm{CCD}$ camera (Image intensifier unit C8600) in vivo. (A, F) Bright field image. (B, G) Bioluminescence detection (no filter). (C, H) Bioluminescence detection with a 520-600 $\mathrm{nm}$ band pass filter (HQ560/80 m). (D, I) Bioluminescence detection with a $607-682 \mathrm{~nm}$ band pass filter (HQ645/75 m). (E, J) Mature leaves of Arabidopsis (E) and tobacco $(\mathrm{J})$ were bombarded with equal amount of $35 \mathrm{~S}::$ CBG68luc or $35 \mathrm{~S}::$ CBRluc and $35 \mathrm{~S}::$ hRluc plasmids. After incubation for $6 \mathrm{~h}$, enzymatic activities were measured by DLRA. Means and SDs are shown $(n=3)$. Asterisks indicate that the CBG68luc luminescence activity is significantly different from that of the CBRluc by Student's $t$-test $(P<0.01)$.

system (Bio-Rad). Aliquots ( $25 \mu \mathrm{l})$ of gold microcarrier $(1.6 \mu \mathrm{m})$ coated with $1 \mu \mathrm{g}$ of $35 \mathrm{~S}::$ CBG68luc or $35 \mathrm{~S}::$ CBRluc in addition to $1 \mu \mathrm{g}$ of $35 \mathrm{~S}::$ hRluc were spread onto macrocarriers. Samples were placed $9 \mathrm{~cm}$ from the stopping screen and bombarded as described previously (Ogura et al. 2005). After bombardment, the samples were incubated for about $6 \mathrm{~h}$ at $22^{\circ} \mathrm{C}$ for Arabidopsis and $26^{\circ} \mathrm{C}$ for tobacco under continuous 
light, and then the luciferase luminescent activity was measured by DLRA. Each sample was transferred into a $2 \mathrm{ml}$ Eppendorf microfuge tube containing $200 \mu \mathrm{l}$ of $1 \times$ passive lysis buffer (Promega) cooled on ice; a $5 \mathrm{~mm}$ zirconia ball was added, and the mixture was disrupted using a TissueLyser (QIAGEN, Valencia, CA) for $1 \mathrm{~min}$ at $25 \mathrm{~Hz}$, then centrifuged at $11,000 \mathrm{~g}$ for $1 \mathrm{~min}$ at $4^{\circ} \mathrm{C}$. For the detection of CBG68luc or CBRluc luminescent activity, $8 \mu \mathrm{l}$ of the supernatant was transferred into a new $1.5 \mathrm{ml}$ microfuge tube containing $40 \mu \mathrm{l}$ LARII solution (Promega), mixed by vortexing, and then placed in a Luminescencer-Octa luminometer (ATTO, Tokyo, Japan). Then, $40 \mu 1$ of Stop and Glo solution (Promega) was added to the reaction mixture for the detection of hRluc luminescent activity. The luminescent activity of CBG68luc was about 4-fold higher than the luminescent activity of CBRluc in Arabidopsis, and about two-fold higher in tobacco (Figure 1E, J). These results are consistent with the in vivo bioluminescence assay of transgenic plants (Figure 1B, G). Together with the previous data (Ogura et al. 2005), it is clear that the luminescence activity of CBG68luc and CBRluc shows some species specificity.

Next, we investigated the emission spectra of CBG68luc and CBRluc from various plant tissues and cells and compared them with the spectra from E. coli. The CaMV 35S promoter is active and DCRA can be used in E. coli (Almond et al. 2003; Assaad and Signer 1990), so we chose to use live cells harboring $35 \mathrm{~S}::$ CBG68luc or $35 \mathrm{~S}::$ CBRluc plasmids. To prepare E. coli samples, a single colony was picked from the LBampicillin plate and cultured in LB medium with ampicillin $\left(50 \mu \mathrm{g} \mathrm{ml}^{-1}\right)$ for $16 \mathrm{~h}$. For the analysis of both transgenic Arabidopsis and tobacco, a seedling was sprayed with $0.2 \mathrm{mM}$ D-luciferin. Transient assays for bioluminescence detection in tobacco BY-2, onion, spinach and lettuce were carried out as described above, except using $2 \mu \mathrm{g}$ plasmid DNA for each assay. Six hours
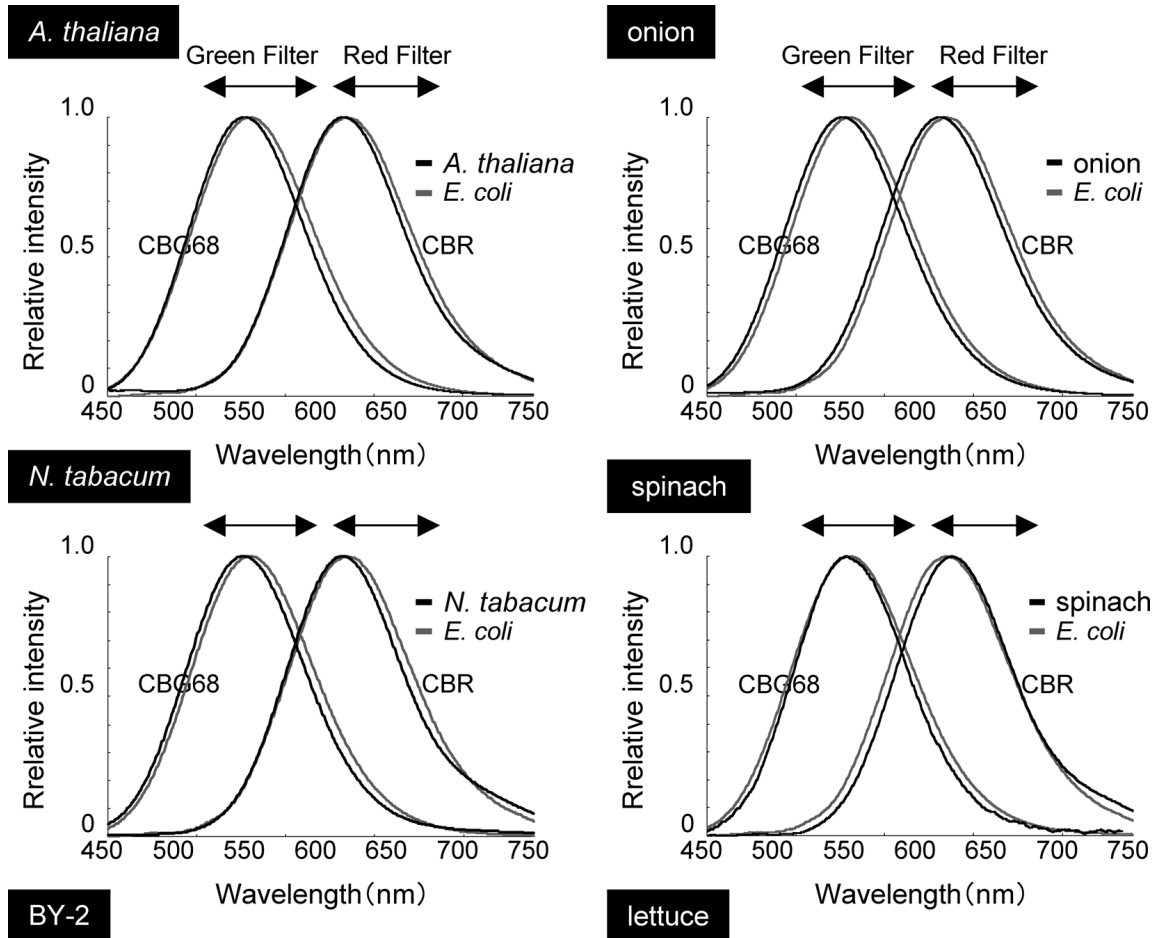

spinach
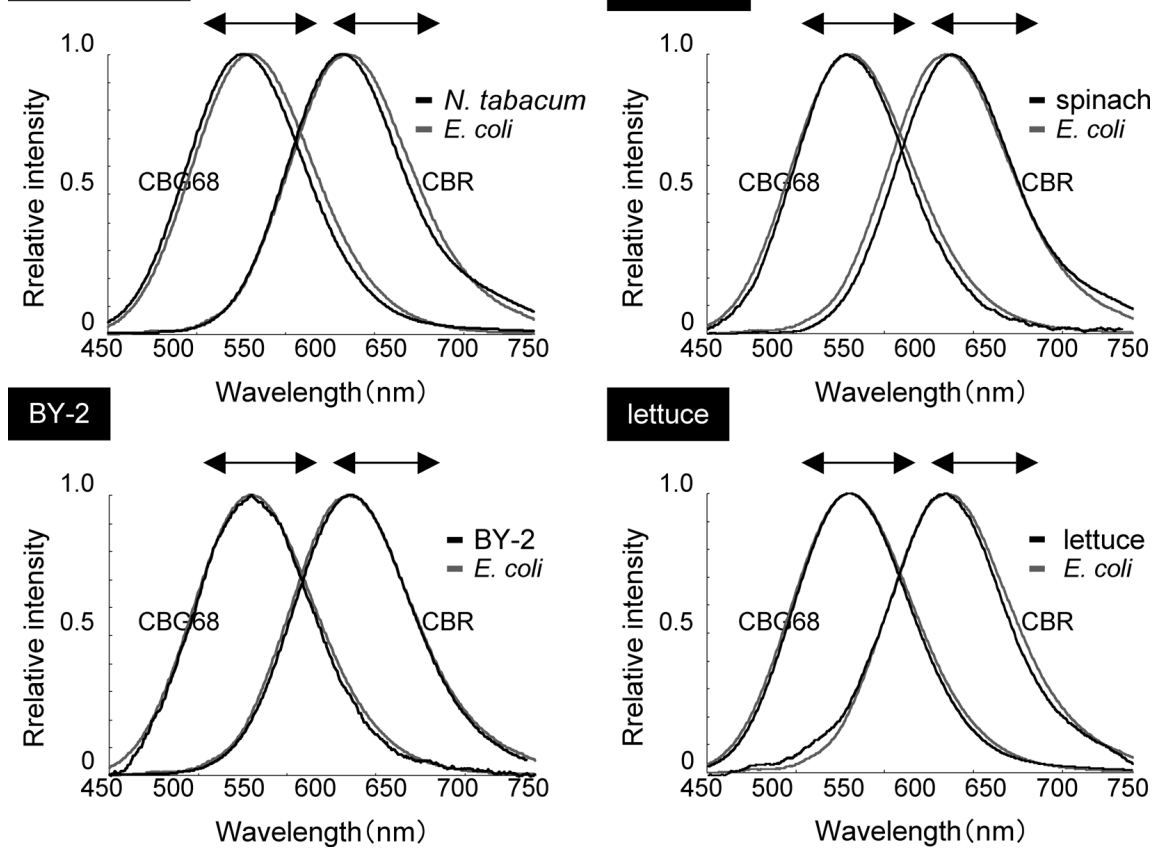

Figure 2. Measurement of emission spectra from E. coli and various plants. The vertical axis shows the relative luminescence intensity and the horizontal axis shows the emission wavelength. The gray lines show emission spectra from E. coli. The black lines show emission spectra from plants. 
after the bombardment, each sample was sprayed with $0.2 \mathrm{mM}$ D-luciferin. The samples were left in the dark for $10 \mathrm{~min}$ to dark-adapt before analysis. The emission spectra were obtained and analyzed by an AB-1850 LumiFL-Spectrocapture spectrometer (ATTO).

As shown in Figure 2, data obtained in this study revealed that the luminescence spectra of CBG68luc and CBRluc from various plant tissues exhibit almost the same pattern as that from E. coli cells. Although plant tissues contain various pigments and autofluorescent materials, these data suggest that the presence of these potentially interfering factors in plant cells and tissues does not seriously influence dual-color bioluminescence detection using optical filters. However, judging from the spectra obtained in this study, the use of a band pass filter for shorter wavelength region is preferable to obtain better separation of the green luminescence.

We concluded that the DCRA system is applicable to monitor regulation of gene expression in plants. However, optimization of assay conditions and optical filter design may be necessary to improve the accuracy and level of detection when monitoring gene expression in vivo using DCRA.

\section{Acknowledgements}

This study was supported in part by a grant from the Development of Fundamental Technologies for Production of High-value Materials using Transgenic Plants (2006-2010) project of the Ministry of Economy, Trade and Industry (METI) of Japan.

\section{References}

Almond B, Hawkins E, Stecha P, Garvin D, Paguio A, Butler B, Beck M, Wood M, Wood KV (2003) A new luminescence: Not your average click beetle. Promega Notes 85: 11-14
Assaad FF, Signer ER (1990) Cauliflower mosaic virus P35S promoter activity in Escherichia coli. Mol Gen Genet 223: $517-520$

Gammon ST, Leevy WM, Gross S, Gokel GW, Piwnica-Worms D (2006) Spectral unmixing of multicolored bioluminescence emitted from heterogeneous biological sources. Anal Chem 78: $1520-1527$

Ikeda M, Ohmiya Y (2005) Multicolor luciferase assay system: one-step monitoring of multiple gene expressions with a single substrate. Biotechniques 38: 891-894

Matsuo N, Minami M, Maeda T, Hiratsuka K (2001) Dual luciferase assay for monitoring transient gene expression in higher plants. Plant Biotechnol 18: 71-75

Murashige T, Skoog F (1962) A revised medium for rapid growth and bioassays with tobacco tissue cultures. Physiol Plant 15: 473-497

Nakajima Y, Kimura T, Suzuki C, Ohmiya Y (2004a) Improved expression of novel red- and green-emitting luciferases of Phrixothrix railroad worms in mammalian cells. Biosci Biotechnol Biochem 68: 948-951

Nakajima Y, Ikeda M, Kimura T, Honma S, Ohmiya Y, Honma K (2004b) Bidirectional role of orphan nuclear receptor RORalpha in clock gene transcriptions demonstrated by a novel reporter assay system. FEBS Lett 565: 122-126

Nakajima Y, Ohmiya Y (2010) Bioluminescence assays: multicolor luciferase assay, secreted luciferase assay and imaging luciferase assay Expert Opin Drug Discov 5: 836-849

Naylor LH (1999) Reporter gene technology: the future looks bright. Biochem Pharmacol 58: 749-757

Ogura R, Matsuo N, Wako N, Tanaka T, Ono S, and Hiratsuka K (2005) Multi-color luciferases as reporters for monitoring transient gene expression in higher plants. Plant Biotechnol 22: $151-155$

Ono S, Tanaka T, Watakabe Y, Hiratsuka K (2004) Transient assay system for the analysis of PR-1a gene promoter in tobacco BY-2 Cells. Biosci Biotechnol Biochem 68: 803-807

Sherf BA, Navarro SL, Rita RH, Wood KV (1996) Dual luciferase reporter assay: An advanced co-reporter technology integrating firefly and renilla luciferase assays. Promega Notes 57: 2-8 